\title{
Desenvolvimento de um Aplicativo Lúdico para Pessoas com Deficiência Intelectual
}

\author{
Leandro Ribeiro Galvão ${ }^{1}$, Edwaldo Soares Rodrigues ${ }^{1}$, Luciano Ribeiro Galvão ${ }^{2}$ \\ ${ }^{1}$ Universidade do Estado de Minas Gerais (UEMG) \\ ${ }^{2}$ Universidade Federal de Lavras (UFLA) \\ \{leandro.galvao, edwaldo.rodrigues\}@uemg.br, lrgalvao@posgrad.ufla.br
}

\begin{abstract}
Intellectual disability affects thousands of people in Brazil. Intending to assist in the care and development of these people, ONGs (non-governmental organizations) and Associations present themselves everywhere in the country. These institutions focus on the educational development of people with disability, since the teaching public system is not prepared to receive them. However, in institutions, there are short-term educational activities, which leave students idle throughout a significant part of the day. Aiming to reduce the idle time of people with disability, this work has developed a ludic mobile application. This application intends to keep them entertained and, at the same time, stimulate logical reasoning. In order to guide the development of the work, it was used the Agile XP development methodology, which contributed to identify scenarios in the application. The developed application is compounded by four modules, and the validation procedure of requirements was made by professionals and students of APAE in Divinopolis city-MG. Validation results has shown that, considering the goals, the application was successful, offering a possibility of recreation and entertainment for intellectually disabled people.
\end{abstract}

Resumo. A deficiência intelectual afeta milhares de pessoas no Brasil. Com o intuito de auxiliar no cuidado e desenvolvimento dessas pessoas, as ONGs e Associações se apresentam por todo país. O foco dessas instituições é o desenvolvimento educacional dos deficientes, visto a falta de preparo da rede pública de ensino em recebê-los. No entanto, nas instituições, as atividades educacionais são de curto prazo, deixando os alunos ociosos durante uma fração significativa do dia. Com o intuito de reduzir o tempo de ociosidade de pessoas com deficiência intelectual, foi desenvolvida, neste trabalho, uma aplicação móvel lúdica. A aplicação tem o intuito de mantê-los entretidos e, ao mesmo tempo, estimular o raciocínio lógico. Para delinear o desenvolvimento do trabalho, foi utilizada a metodologia de desenvolvimento Ágil XP, que contribuiu na identificação dos cenários presentes na aplicação. A aplicação desenvolvida é composta por quatro módulos, e o processo de validação dos requisitos foi feito por profissionais e alunos da APAE da cidade de Divinópolis-MG. Os resultados da validação mostram que a aplicação obteve êxito em relação aos objetivos traçados, oferecendo, assim, uma possibilidade de lazer e entretenimento para deficientes intelectuais. 
VII Congresso Brasileiro de Informática na Educação (CBIE 2018)

Anais do XXIX Simpósio Brasileiro de Informática na Educação (SBIE 2018)

\section{Introdução}

A deficiência intelectual é uma condição que afeta milhares de pessoas no Brasil. O deficiente intelectual possui dificuldades para resolver problemas, assimilar ideias abstratas, metáforas, concepção de tempo, valores monetários, bem como manter relações sociais, entender determinados aspectos cognitivos, seguir regras e praticar muitas atividades comuns do dia a dia. Segundo a American Association on Intellectual and Developmental Disabilities [AAIDD 2018], a deficiência intelectual se caracteriza como uma limitação na execução de atividades cotidianas devido ao funcionamento intelectual inferior à média da população.

Devido a pouca assistência do estado na inserção de pessoas com deficiência intelectual, associações e ONGs acabam, dentro das suas possibilidades, exercendo um papel importante na socialização de tais pessoas. Dentre as ONGs, destacam-se as Associações de Pais e Amigos dos Excepcionais (APAEs). A APAE tem como objetivo auxiliar pessoas com deficiências diversas, a exemplo da deficiência intelectual, prestando assistência de natureza associativa, filantrópica, promocional, educacional, terapêutica, profissional, cultural, esportiva e social.

No entanto, apesar do apoio das associações e ONGs aos deficientes intelectuais, há uma situação em que elas pouco podem oferecer. Seu inestimável auxílio fica restrito somente ao tempo de presença dos deficientes na instituição, já que, em boa parte do tempo, os indivíduos encontram-se em suas respectivas residências. Nesse contexto, um ponto crucial gira em torno de como os deficientes mantêm relações pessoais em seus lares e como conseguem ter algum tipo de lazer. De acordo com [Cia et al. 2006], deficientes cujos pais procuram manter um diálogo e proporcionar-lhes lazer e entretenimento possuem um melhor rendimento educacional, já que situações interpessoais ocorrem em diversas contextos, tais como o familiar, o de lazer, dentre outros.

Deste modo, verifica-se a necessidade da presença dos pais e do estímulo dos deficientes às atividades de lazer e entretenimento. No entanto, proporcionar lazer e entretenimento aos deficientes não é uma tarefa tão trivial, por isso, muitos pais simplesmente optam por deixar seus filhos assistirem a programas televisivos. Essa opção pode não ser a melhor, já que, de certo modo, a partir de determinado momento, tais programas se tornarão parte da rotina e não mais farão com que os deficientes tenham algum tipo de lazer, além de nada agregar ao seu processo de desenvolvimento intelectual.

Nesse contexto, este trabalho tem por objetivo fornecer uma possibilidade adicional de entretenimento e lazer aos deficientes intelectuais, usando da ludicidade e aliando, de modo superficial, conceitos relacionados à capacitação dos deficientes para o mercado de trabalho, auxiliando-os em atividades rotineiras, simples e importantes, como, por exemplo: saudar as pessoas da maneira correta, consultar o calendário e relógio para aumentar a sua noção de tempo. Para isso, foi desenvolvida uma aplicação destinada a aplicativos móveis, pois estes são mais fáceis de serem utilizados pelos deficientes, uma vez que a coordenação motora é facilitada em relação ao uso de computadores. Uma das vantagens do aplicativo desenvolvido consiste na possibilidade de o deficiente conseguir se entreter em momentos de tempo ocioso, sendo uma necessidade visualizada atualmente.

Este artigo está organizado da seguinte maneira: na Seção 2, são discutidos os tra- 
VII Congresso Brasileiro de Informática na Educação (CBIE 2018)

Anais do XXIX Simpósio Brasileiro de Informática na Educação (SBIE 2018)

balhos relacionados; a Seção 3 apresenta a metodologia de desenvolvimento da aplicação; na Seção 4, são apresentados os cenários presentes na aplicação; a Seção 5 apresenta a aplicação aqui denominada "Socializar", ilustrando algumas das telas presentes no aplicativo; na Seção 6, é verificada a validação da aplicação e apresentados os principais resultados encontrados; por fim, na Seção 7, são apresentadas as considerações finais do trabalho, bem como sugestões de trabalhos futuros.

\section{Trabalhos Relacionados}

Diversos trabalhos foram propostos na literatura para auxiliar no desenvolvimento cognitivo de pessoas com necessidades especiais. A grande maioria desses trabalhos lida com o processo de alfabetização.

No trabalho de [Rodrigues et al. 2011], foi desenvolvida uma plataforma lúdica para pessoas com deficiência intelectual. A plataforma é composta por jogos que auxiliam no processo de aprendizagem do público-alvo. O objetivo principal deste trabalho é auxiliar diretamente nas dificuldades do usuário em assimilar informações. Os autores implantaram o aplicativo na Associação de Pais e Amigos dos Excepcionais (APAE) da cidade de Arcos-MG e obtiveram bons resultados em relação à adesão dos usuários e também no processo de aprendizagem dos mesmos.

Em [Barbosa and Dantas 2014] foram utilizados jogos do sistema operacional Pandorga ${ }^{1}$ durante a prática de informática de alunos com Necessidades Especiais (NEE) na APAE da cidade de Patos - PB. O sistema operacional Pandorga é uma distribuição Linux voltada especialmente para a educação de crianças e pré-adolescentes. Os experimentos evidenciaram a importância da aplicação de jogos educacionais no processo de aprendizagem de alunos com NEE.

No trabalho de [Ferreira 2016], foi proposta uma ferramenta para educação, com foco na alfabetização de crianças com síndrome de Down. A ferramenta possui atividades que praticam a pronúncia, escrita e identificação de letras do alfabeto. Esse trabalho contou com o apoio de pedagogos e fonoaudiólogos, sendo que sua implantação ocorreu em uma escola no interior da Paraíba. Os autores concluíram que o processo de aprendizagem por meio de técnicas tradicionais é muito mais lento que a utilização da ferramenta proposta.

O apoio à alfabetização de adultos e crianças com deficiência intelectual foi o foco do trabalho proposto em [Veneziano et al. 2013]. Nesse trabalho, foi disponibilizado um software implementado em Flash, uma plataforma de desenvolvimento muito utilizada em aplicações Web. O software proposto pelos autores é denominado "Participar" e exercita a pronúncia, a escrita e a identificação das letras do alfabeto. Os autores validaram a aplicação em escolas públicas do Distrito Federal e concluíram que os recursos multimídias disponibilizados na aplicação contribuíram para o sucesso da etapa de validação.

O processo de alfabetização de pessoas com deficiência intelectual tem sido frequentemente tema de pesquisa, no entanto, é importante ressaltar a falta de trabalhos abordando a utilização de tecnologias móveis por deficientes intelectuais. Além disso, outro fator que motiva a utilização de smartphones e tablets é o fato dos mesmos possuírem telas sensíveis ao toque, sendo fácil a interação quando comparado ao teclado e

\footnotetext{
${ }^{1}$ https://lief.if.ufrgs.br/pub/pandorga
} 
VII Congresso Brasileiro de Informática na Educação (CBIE 2018)

Anais do XXIX Simpósio Brasileiro de Informática na Educação (SBIE 2018)

mouse de um computador.

\section{Desenvolvimento Ágil}

A metodologia de desenvolvimento ágil de software foi adotada neste trabalho para desenvolver a aplicação Socializar. Dentre os diversos métodos ágeis existentes na literatura, destaca-se o eXtreme Programming (XP) devido a sua facilidade de implementação e alta adesão pelas equipes de desenvolvimento.

A utilização do XP é recomendada para projetos de pequeno a médio porte, cujos requisitos são complexos e de difícil elicitação [Teles 2017]. Por meio da elaboração de cenários descritos pelo cliente, versões do software são obtidas. O processo de validação das versões do software ocorre logo após o seu desenvolvimento e busca validar a implementação dos requisitos elicitados.

O XP foi adotado neste trabalho devido ao tamanho da aplicação e à complexidade dos requisitos, visto que o público-alvo possui necessidades especiais. Os pedagogos, psicólogos, assistentes sociais, professores e administradores da APAE foram consultados na fase de elaboração dos cenários. Os requisitos obtidos focaram na prática de atividades lúdicas utilizando tecnologias móveis por pessoas com deficiência intelectual.

\section{Cenários}

A construção dos cenários ocorreu por meio de visitas à Associação de Pais e Amigos dos Excepcionais (APAE) da cidade de Divinópolis-MG. Durante essas visitas, foi possível elaborar diversos cenários, visto a carência dos alunos da APAE em relação ao uso de tecnologias.

Os cenários são compostos por duas partes, sendo a primeira composta por uma narrativa e a segunda por tópicos. A primeira parte descreve o resultado das entrevistas feitas com os funcionários da APAE. Já na segunda parte, são apresentados tópicos elaborados pelos autores deste trabalho que fazem parte dos requisitos da aplicação. A seguir serão apresentados os cenários elaborados.

\subsection{Saudações}

A interação social da pessoa com deficiência intelectual deve ser estimulada. Normalmente, o primeiro contato entre duas pessoas ocorre por meio de uma saudação. Desta forma, a criação de uma atividade lúdica que estimule saudações é muito importante.

I Criação de atividades contendo as saudações "bom dia", "boa tarde" e "boa noite".

II Inserir imagens ilustrativas.

III Motivar o usuário por meio de mensagens de incentivo e apoio.

\subsection{Identificar o dia e a hora}

Os alunos da associação possuem dificuldades em identificar a hora utilizando um relógio. Além disso, a afinidade deles com o uso do calendário é ainda menor do que com o relógio.

I As atividades devem apresentar imagens de relógios analógicos e digitais.

II Devido à baixa familiaridade dos usuários com o calendário, a atividade envolvendo o mesmo deve ter grau de complexidade inferior às demais atividades.

III A cada tentativa incorreta do usuário, o grau de complexidade da atividade deverá ser reduzido automaticamente. 
VII Congresso Brasileiro de Informática na Educação (CBIE 2018)

Anais do XXIX Simpósio Brasileiro de Informática na Educação (SBIE 2018)

\subsection{Raciocínio Lógico}

Os familiares dos alunos da associação reclamam da falta de atividades lúdicas disponíveis fora da associação. Devido à falta de atividades adequadas, os pais dos deficientes intelectuais fornecem atividades infantis para seus filhos.

I As atividades devem estimular o raciocínio lógico.

II O uso de animações não é recomendado, pois o deficiente intelectual apresenta alto grau de déficit de atenção.

III Todas as palavras devem utilizar fonte Arial e letras maiúsculas para facilitar a leitura. A escolha da fonte se deve ao fato de a mesma possuir poucos adornos e ser utilizada no processo de alfabetização dos alunos.

\section{Aplicação Socializar}

A implementação da aplicação Socializar adotou o framework PhoneGap. A aplicação e o código-fonte estão disponíveis no GitHub ${ }^{2}$. O PhoneGap é um framework de desenvolvimento web híbrido que utiliza as linguagens web HTML5, CSS e Javascript ao invés das linguagens nativas das diversas plataformas móveis [Lopes 2016]. Desta forma, a aplicação gerada é compatível com as plataformas Android, IOS, Windows Phone, entre outras.

O desenvolvimento da aplicação foi guiado pelos cenários descritos na Seção 4. Com base nesses cenários, foram implementados 4 módulos interativos. Cada módulo possui como foco a prática de uma tarefa específica, definida no respectivo cenário.

Dentre os requisitos obtidos na elaboração dos cenários, alguns foram implementados em todos os módulos devido a sua importância. Um exemplo de requisito implementado de forma global é a apresentação de mensagens de incentivo e apoio após a realização de uma atividade.

\subsection{Módulo 1}

O objetivo principal do primeiro módulo é a prática de cumprimentos e saudações comumente utilizadas no cotidiano. Nesse módulo, foram utilizados poucos elementos de distração para garantir o máximo de foco na atividade principal do módulo. A utilização de telas com pouca informação é importante independente da capacidade cognitiva do usuário, pois, quanto maior a quantidade de informação, maior é a chance do usuário se dispersar do objetivo principal [Galitz 2007].

O módulo 1 apresenta, em sua primeira tela, um exercício envolvendo o cumprimento "olá". Nessa atividade, o usuário deve organizar as letras para que a palavra "olá" seja formada. A primeira atividade desse módulo está presente na Figura 1 (a), na qual é possível observar a implementação de requisitos globais como o uso de letras maiúsculas e número reduzido de elementos na tela.

A segunda tela do módulo 1 exercita a prática de saudações tais como "bom dia", "boa tarde" e "boa noite", conforme ilustrado na Figura 1 (b). É importante destacar que, quando uma opção incorreta é selecionada, a mesma é removida da tela e a chance de acerto é elevada, motivando o usuário a tentar novamente.

\footnotetext{
${ }^{2}$ https://github.com/leandrodvmg/Socializar
} 
VII Congresso Brasileiro de Informática na Educação (CBIE 2018)

Anais do XXIX Simpósio Brasileiro de Informática na Educação (SBIE 2018)

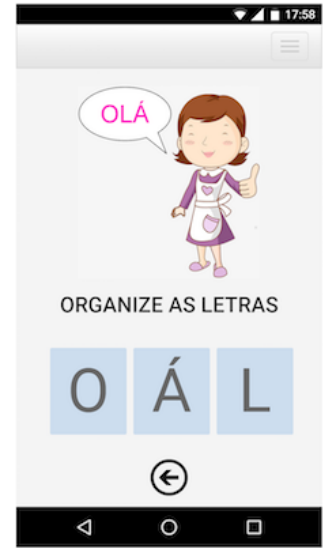

(a)

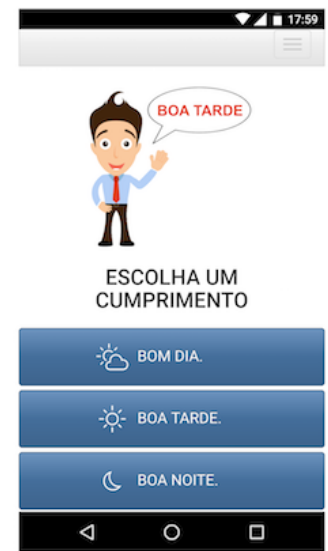

(b)

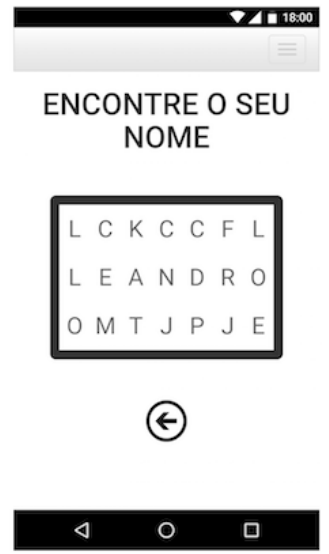

(c)

Figura 1. Atividades do módulo 1 da aplicação Socializar: (a) ordenar as letras; (b) escolher uma saudação; (c) encontrar o próprio nome.

A última tela do módulo 1 apresenta um caça-palavras simplificado. O primeiro nome do usuário, requisitado logo que a aplicação é iniciada, deve ser encontrado em uma das três linhas do caça-palavras. O nome sempre será apresentado de acordo com a seguinte direcionalidade: escrita horizontal e começando da esquerda para direita, assim como na Figura 1 (c).

\subsection{Módulo 2}

O módulo 2 é composto por duas atividades envolvendo o relógio ou o calendário. $\mathrm{O}$ objetivo principal desse módulo é praticar a identificação do dia e da hora. O grau de complexidade desse módulo é superior ao módulo 1 devido à utilização de objetos que requerem um esforço cognitivo maior.

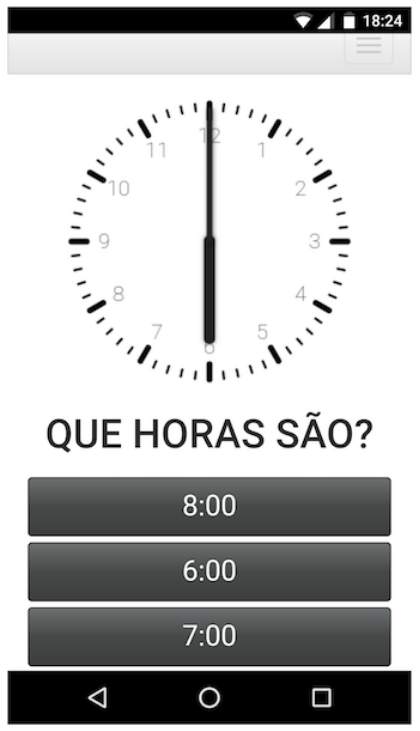

(a)

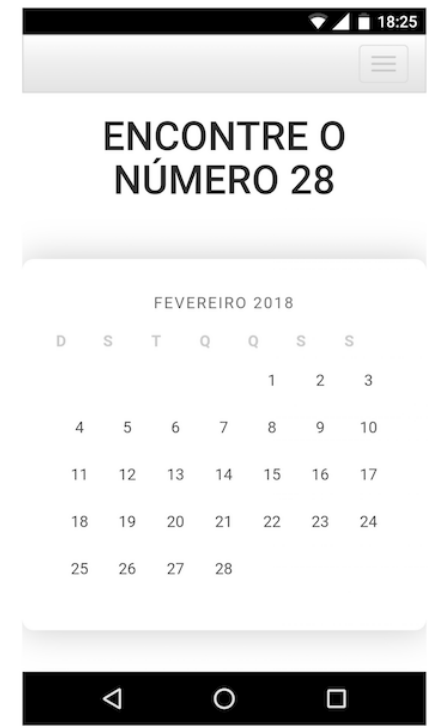

(b)

Figura 2. Atividades do módulo 2 da aplicação Socializar: (a) informar a hora; (b) encontrar o número. 
VII Congresso Brasileiro de Informática na Educação (CBIE 2018)

Anais do XXIX Simpósio Brasileiro de Informática na Educação (SBIE 2018)

O módulo 2 apresenta, em sua tela inicial, a imagem de um relógio analógico. Nessa atividade, o usuário deve informar a hora definida no relógio, assim como está presente na Figura 2 (a). Devido a pouca afinidade do público-alvo com o relógio, foi configurado para que a atividade exija que o usuário saiba identificar apenas a hora do relógio.

A última tela do módulo 2 exercita a identificação do dia no calendário, conforme ilustrado na Figura 2 (b). O calendário possui mais elementos de distração do que o relógio, por isso essa atividade é considerada mais complexa do que a anterior. No entanto, a identificação do mês e o respectivo dia da semana não são necessários.

\subsection{Módulo 3}

Diferente dos módulos 1 e 2, no módulo 3 é apresentado apenas uma atividade contendo três telas distintas. Nesse módulo, busca-se o desenvolvimento do raciocínio lógico do usuário. A atividade desenvolvida nesse módulo também tem como objetivo entreter os deficientes intelectuais.

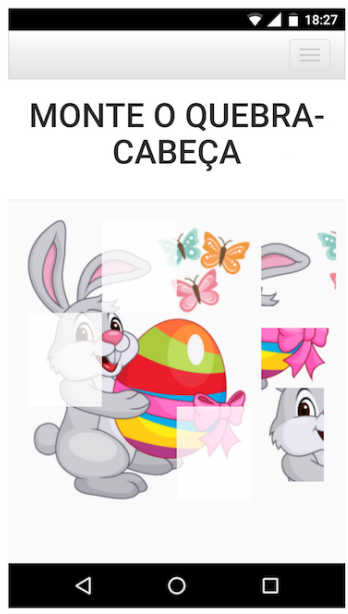

(a)
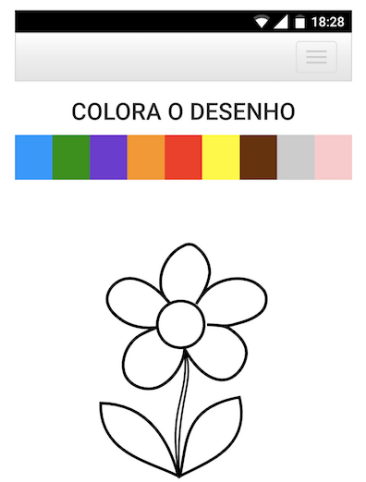

PRÓXIMO

(b)

Figura 3. Atividades do módulo 3 e 4 da aplicação Socializar: (a) quebra-cabeça do módulo 3; (b) desenho para colorir do módulo 4.

As telas do módulo 3 apresentam diferentes quebra-cabeças. As imagens utilizadas na construção da atividade remetem a feriados do dia das mães, carnaval e páscoa. A adoção de quebra-cabeças temáticos ocorre devido à grande afinidade do público-alvo com datas comemorativas, uma vez que são frequentemente tema de atividades educacionais. Um exemplo de quebra-cabeça disponível no módulo 3 está presente na Figura 3 (a).

\subsection{Módulo 4}

O objetivo principal do módulo 4 é desenvolver a afinidade do usuário com o dispositivo móvel por meio de uma atividade lúdica. A atividade presente no módulo 4 é composta por quatro telas distintas.

As telas do módulo 4 apresentam imagens contendo desenhos para colorir. Além das imagens, cada tela possui também uma palheta de cores, na qual o usuário escolhe a 
VII Congresso Brasileiro de Informática na Educação (CBIE 2018)

Anais do XXIX Simpósio Brasileiro de Informática na Educação (SBIE 2018)

cor que quer utilizar. Um exemplo de desenho para colorir disponível no módulo 4 está presente na Figura 3 (b).

\section{Validação e Resultados}

O processo de validação da aplicação Socializar foi feita por meio de protótipos, seguindo os princípios do desenvolvimento ágil descritos na Seção 3. Cada cenário elaborado, em conjunto com os profissionais da APAE Divinópolis-MG, originou um protótipo distinto.

Os protótipos foram avaliados inicialmente pela equipe da associação antes de serem disponibilizados para os alunos. As avaliações dos protótipos utilizaram um tablet Samsung Galaxy Tab E com Tela de 9,6 polegadas da própria associação.

Um grupo composto por 10 alunos da APAE Divinópolis-MG foi adotado no processo de validação dos protótipos. Os participantes possuem diferentes idades e níveis de deficiência intelectual, retratando assim a realidade da associação. Dentre os alunos participantes, apenas um não compareceu a todos os dias de testes.

A avaliação dos protótipos dos módulos 1 e 2 mostrou que os requisitos descritos nos respectivos cenários foram implementados corretamente. Os alunos da associação não tiveram dificuldades em concluir as atividades disponíveis nesses módulos. No entanto, foi observado grande apatia e desinteresse dos alunos durante os testes. Ao final dos testes, foram feitas alguns perguntas aos alunos e percebeu-se que, apesar de atender aos requisitos, os módulos 1 e 2 não proporcionaram uma forma de entretenimento para os participantes.

De posse dos resultados da avaliação dos protótipos dos módulos 1 e 2 , foi incluído nos requisitos dos módulos 3 e 4 a necessidade de entreter o usuário durante a realização das atividades. Com base nesse novo requisito, os protótipos dos módulos 3 e 4 foram avaliados e observou-se grande interesse durante o desenvolvimento das atividades. É importante ressaltar que todos os alunos que participaram dos testes completaram as atividades dos módulos 3 e 4 repetidas vezes, o que não aconteceu nos dois primeiros módulos devido ao baixo interesse dos participantes.

\section{Considerações Finais}

No Brasil, milhares de jovens e adultos possuem algum tipo de deficiência intelectual. Apesar disso, o número de softwares desenvolvido para esse grupo é muito pequeno. A grande maioria das pesquisas relacionadas a esse tema foca na alfabetização de deficientes intelectuais.

Existe uma carência de softwares que busquem, de forma lúdica, aumentar a afinidade do deficiente intelectual com novas tecnologias, como, por exemplo, os dispositivos móveis. Neste trabalho, é apresentado uma aplicação denominada "Socializar", que busca desenvolver as habilidades do usuário na utilização de smartphones, tablets, entre outros dispositivos móveis de forma lúdica.

O levantamento de requisitos e a validação da aplicação Socializar ocorreram na Associação de Pais e Amigos dos Excepcionais (APAE) da cidade de Divinópolis-MG. A APAE é uma instituição não governamental filantrópica presente em diversas cidades do Brasil. A associação oferece assistência médica e pedagógica para pessoas com autismo, síndrome de Down, deficiência múltipla, entre outros casos. 
VII Congresso Brasileiro de Informática na Educação (CBIE 2018)

Anais do XXIX Simpósio Brasileiro de Informática na Educação (SBIE 2018)

Os testes de validação da aplicação mostraram que, apesar da implementação dos requisitos iniciais ter sido correta, era necessário que outros requisitos fossem implementados para garantir a adesão do público-alvo. Com isso, modificações foram feitas para que os jovens e adultos com deficiência intelectual se divertissem ao utilizar a aplicação.

Portanto, este trabalho disponibiliza uma aplicação que pode ser utilizada em dispositivos móveis que utilizem diferentes plataformas. É importante ressaltar que a interação de pessoas com deficiência intelectual por meio do toque na tela é mais fácil do que utilizando periféricos como teclado e mouse. Por esse motivo, apesar dos diferentes níveis de deficiência intelectual existente no grupo de teste, todos os usuários conseguiram completar as atividades.

Como trabalhos futuros, os autores deste trabalho pretendem implementar novos módulos e expandir os testes com o usuário, de modo a ter avaliações mais precisas. Além disso, a incorporação do Método de Avaliação da Comunicabilidade (MAC) na fase de validação da aplicação pode gerar resultados ainda melhores.

\section{Referências}

AAIDD (2018). American association on intellectual and developmental disabilities, acessado em: 21/05/2018. URL: https://aaidd.org/intellectualdisability/definition/faqs-on-intellectual-disability.

Barbosa, C. L. and Dantas, R. F. (2014). Estudo sobre a aplicação de jogos educativos de computador no processo de ensino-aprendizagem de crianças com necessidades educacionais especiais. Congresso Nacional de Educação.

Cia, F., de Sousa Pereira, C., Pereira Del Prette, Z. A., and Del Prette, A. (2006). Habilidades sociais parentais e o relacionamento entre pais e filho. Psicologia em estudo, 11(1):73-81.

Ferreira, J. S. (2016). Produção de um protótipo intuitivo para auxiliar na alfabetização de alunos com síndrome de down. Monografia; Universidade Estadual da Paraíba UEPB.

Galitz, W. O. (2007). The essential guide to user interface design: an introduction to GUI design principles and techniques. John Wiley \& Sons.

Lopes, S. (2016). Aplicações mobile híbridas com Cordova e PhoneGap. Editora Casa do Código.

Rodrigues, E. S., Oliveira, F. M., Noberto, A. P., and Silva, G. J. (2011). Estudo de campo para o desenvolvimento de um aplicativo computacional lúdico auxiliar ao processo de ensino aprendizagem de deficientes intelectuais da apae, arcos-mg. Belo Horizonte: PUC. Atas do VI Seminário de Extensão da PUC Minas.

Teles, V. M. (2017). Extreme Programming: Aprenda como encantar seus usuários desenvolvendo software com agilidade e alta qualidade. Novatec Editora.

Veneziano, W. H., Pereira, M. H. B. E., Freire, T. G. M., and Silva, R. D. (2013). Programa participar: Software educacional de apoio à alfabetização de jovens e adultos com deficiência intelectual. In Brazilian Symposium on Computers in Education (Simpósio Brasileiro de Informática na Educação-SBIE), volume 24, page 477. 